\title{
Expansion of the density: a Wiener-chaos approach
}

\author{
DAVID MÁRQUEZ-CARRERAS* and MARTA SANZ-SOLÉ** \\ Facultat de Matemàtiques, Universitat de Barcelona, Gran Via 585, 08007 Barcelona, Spain \\ e-mail:*marquez@cerber.mat.ub.es**sanz@cerber.mat.ub.es
}

\begin{abstract}
We prove a Taylor expansion of the density $p^{\varepsilon}(y)$ of a Wiener functional $F^{\varepsilon}$ with Wiener-chaos decomposition $F^{\varepsilon}=y+\sum_{n=1}^{\infty} \varepsilon^{n} I_{n}\left(f_{n}\right), \varepsilon \in(0,1]$. Using Malliavin calculus, a precise description of the coefficients in the development in terms of the multiple integrals $I_{n}\left(f_{n}\right)$ is provided. This general result is applied to the study of the density in two examples of hyperbolic stochastic partial differential equations with linear coefficients, where the driving noise has been perturbed by a coefficient $\varepsilon$.
\end{abstract}

Keywords: Malliavin calculus; probability densities; stochastic partial differential equations; Wiener functionals

\section{Introduction}

Let $(T, \mathscr{T}, \mu)$ be an atomless measure space with a $\sigma$-finite measure $\mu$. Set $\mathscr{H}=L^{2}(T, \mathscr{T}, \mu)$ and let $W=\left\{W_{h}, h \in \mathscr{H}\right\}$ be a Gaussian zero-mean process with $\mathrm{E}\left(W_{h}, W_{h^{\prime}}\right)=\left\langle h, h^{\prime}\right\rangle_{\mathscr{H}}$ defined on some probability space $(\Omega, \mathscr{A}, P)$. Let $\mathscr{F}$ be the $\sigma$ field generated by $W$. We consider a measurable mapping $F: \Omega \rightarrow \mathbb{R}^{d}$ belonging to $L^{2}(\Omega, \mathscr{F}, P)$ with Wiener-chaos decomposition $F=\mathrm{E}(F)+\sum_{n=1}^{\infty} I_{n}\left(f_{n}\right)$. Let $\left\{F^{\varepsilon}, \varepsilon \in(0,1]\right\}$ be defined by $F^{\varepsilon}=\mathrm{E}(F)+\sum_{n=1}^{\infty} \varepsilon^{n} I_{n}\left(f_{n}\right)$. We assume that the probability law of each $F^{\varepsilon}$ is absolutely continuous with respect to the Lebesgue measure on $\mathbb{R}^{d}$. The purpose of this paper is to study the Taylor expansion of the density $p^{\varepsilon}(y)$ of the probability law of $F^{\varepsilon}$ at $\varepsilon=0$, where $y=\mathrm{E}(F)=\mathrm{E}\left(F^{\varepsilon}\right)$. A similar problem has been widely studied for diffusion processes (see, for example, Molchanov (1975), Azencott (1984), Bismut (1984) and Ben Arous (1988)) for the family $F^{\varepsilon}, \varepsilon \in(0,1]$, obtained by changing the time $t$ into $\varepsilon t$. In this case, because of the scaling property of the Brownian motion, we obtain by probabilistic methods the behaviour of the density $p_{t}(y)$ of the diffusion $X_{t}$ for small $t$. For general families of Wiener functionals the problem has been addressed by Watanabe (1987) and Takanobu and Watanabe (1993).

Our main goal is to give a precise description of the coefficients of the expansion using the Wiener-chaos decomposition of $F$ and the particular structure of the family $\left\{F^{\varepsilon}, \varepsilon \in(0,1]\right\}$. They correspond to densities of completely explicit Radon measures. First we prove differentiability of the mapping $\varepsilon \mapsto F^{\varepsilon}$ on appropriate derivation spaces related with the Sobolev spaces $\mathbb{D}^{N, 2}$ of Malliavin calculus. The derivatives are expressed in terms of the 
multiple integrals $I_{n}\left(f_{n}\right)$. Then, using the approach of Léandre (1988) and Léandre and Russo (1992), we obtain in Theorem 2.4 the Taylor expansion for the density via the expansion of $f\left(F^{\varepsilon}\right)$, for smooth $f$, and integration by parts. As for diffusions, the odd-order coefficients of the expansion vanish and the non-null coefficients belong to a finite Wiener-chaos diffusion.

The paper is divided into two sections. Section 2 is devoted to the proof of the main result described before; Section 3 contains two examples of hyperbolic stochastic partial differential equations where this result can be applied. As usually, all constants will be denoted by $C$ independent of its value. We refer the reader to Nualart (1995) for the notation and notions on Malliavin calculus used in this paper.

\section{Expansion of the density}

Let $F$ be a $\mathbb{R}^{d}$-valued random vector defined on the abstract Wiener space $(\Omega, \mathscr{H}, P)$, belonging to $L^{2}(\Omega)$. Let $F=\sum_{n=0}^{\infty} I_{n}\left(f_{n}\right)$ be its Wiener-chaos representation. For any $\varepsilon \in(0,1]$ we define

$$
F^{\varepsilon}(\omega)=\sum_{n=0}^{\infty} \varepsilon^{n} I_{n}\left(f_{n}\right)
$$

Clearly, the series defining $F^{\varepsilon}(\omega)$ converges in $L^{2}(\Omega)$. The purpose of this section is to obtain an asymptotic expansion of the density of $F^{\varepsilon}, p^{\varepsilon}(y)$ at $y=\mathrm{E}(F)=\mathrm{E}\left(F^{\varepsilon}\right)$, whenever it exists. We shall follow some ideas introduced by Léandre (1988) (see also Léandre and Russo (1992)). The first result establishes the smoothness of $F^{\varepsilon}$ with respect to $\varepsilon$. To this end we first introduce some derivation spaces, which are related to the classical Sobolev spaces $\mathbb{D}^{k, p}$ of the Malliavin calculus.

For any $j \in \mathbb{Z}^{+}$, set

$$
\Delta^{j, 2}=\left\{F \in L^{2}(\Omega): \sum_{k=j}^{\infty}\left(\frac{k !}{(k-j) !}\right)^{2} k !\left\|f_{k}\right\|_{2}^{2}<\infty\right\},
$$

where $\left\|f_{k}\right\|_{2}$ denotes the norm of $f_{k}$ in $L^{2}\left(T^{k}\right)$. Note that $\Delta^{0,2}=L^{2}(\Omega)$ and $\Delta^{j, 2}$ decreases as $j$ increases.

In the next proposition, $d=1$. For $d>1$ the result applies componentwise.

Proposition 2.1. Fix $j \geqslant 1$, and assume that $F \in \Delta^{j+1,2}$. There exists a version of $\left\{F^{\varepsilon}, \varepsilon \in(0,1)\right\}$ which is of class $\mathscr{C}^{j}$. Moreover,

$$
\frac{\mathrm{d}^{j} F^{\varepsilon}}{\mathrm{d} \varepsilon^{j}}=\sum_{k=j}^{\infty} \frac{k !}{(k-j) !} \varepsilon^{k-j} I_{k}\left(f_{k}\right)
$$

Proof. Consider first the case $j=1$. For $\varepsilon$, $\xi$ with $0<\varepsilon+\xi<\varepsilon_{0}<1$ we have

$$
\frac{F^{\varepsilon+\xi}-F^{\varepsilon}}{\xi}=\left\{\sum_{k=1}^{\infty} \sum_{i=0}^{k-1}\left(\begin{array}{c}
k \\
i
\end{array}\right) \varepsilon^{i} \xi^{k-i} I_{k}\left(f_{k}\right)\right\} / \xi=A_{1}^{\varepsilon}+\xi A_{2}^{\varepsilon, \xi},
$$


with

$$
\begin{aligned}
A_{1}^{\varepsilon} & =\sum_{k=1}^{\infty} k \varepsilon^{k-1} I_{k}\left(f_{k}\right), \\
A_{2}^{\varepsilon, \xi} & =\sum_{k=2}^{\infty} \sum_{i=0}^{k-2}\left(\begin{array}{c}
k \\
i
\end{array}\right) \varepsilon^{i} \xi^{k-i-2} I_{k}\left(f_{k}\right) .
\end{aligned}
$$

Since $F \in \Delta^{1,2}$, the series defining $A_{1}^{\varepsilon}$ converges in $L^{2}(\Omega)$. In addition,

$$
\sup _{\xi}\left|A_{2}^{\varepsilon, \xi}\right| \leqslant C X_{1},
$$

with $X_{1}:=\left[\sum_{k=2}^{\infty} k^{2}(k-1)^{2}\left\{I_{k}\left(f_{k}\right)\right\}^{2}\right]^{1 / 2}$. Indeed, by the Schwarz inequality,

$$
\begin{aligned}
\left|A_{2}^{\varepsilon, \xi}\right| & \leqslant \sum_{k=2}^{\infty} \sum_{i=0}^{k-2}\left(\begin{array}{c}
k-2 \\
i
\end{array}\right) \frac{k(k-1)}{(k-i)(k-i-1)} \varepsilon^{i} \xi^{k-i-2}\left|I_{k}\left(f_{k}\right)\right| \\
& \leqslant \frac{1}{2} \sum_{k=2}^{\infty} k(k-1)(\varepsilon+\xi)^{k-2}\left|I_{k}\left(f_{k}\right)\right| \\
& \leqslant \frac{1}{2}\left(\sum_{k=0}^{\infty}(\varepsilon+\xi)^{2 k}\right)^{1 / 2}\left(\sum_{k=2}^{\infty} k^{2}(k-1)^{2}\left\{I_{k}\left(f_{k}\right)\right\}^{2}\right)^{1 / 2} \\
& \leqslant \frac{1}{2} \frac{1}{\left(1-\varepsilon_{0}^{2}\right)^{1 / 2}} X_{1} .
\end{aligned}
$$

Since $F \in \Delta^{2,2}, X_{1}$ is finite a.s. Consequently, from (2.2) we obtain

$$
\lim _{\xi \rightarrow 0} \frac{F^{\varepsilon+\xi}-F^{\varepsilon}}{\xi}=A_{1}^{\varepsilon}, \quad \text { a.s. }
$$

Let $j>1$ and assume that the statement holds for any $k \in\{1, \ldots, j-1\}$. Set

$$
d_{j-1}^{\varepsilon}=\frac{\mathrm{d}^{j-1} F^{\varepsilon}}{\mathrm{d} \varepsilon^{j-1}}=\sum_{k=j-1}^{\infty} \frac{k !}{(k-j+1) !} \varepsilon^{k-j+1} I_{k}\left(f_{k}\right) .
$$

Then,

$$
\frac{\mathrm{d}_{j-1}^{\varepsilon+\xi}-\mathrm{d}_{j-1}^{\varepsilon}}{\xi}=B_{1}^{\varepsilon}+\xi B_{2}^{\varepsilon, \xi},
$$

with 


$$
\begin{aligned}
B_{1}^{\varepsilon} & =\sum_{k=j}^{\infty} \frac{k !}{(k-j) !} \varepsilon^{k-j} I_{k}\left(f_{k}\right), \\
B_{2}^{\varepsilon, \xi} & =\sum_{k=j+1}^{\infty} \sum_{i=0}^{k-j-1} \frac{k !}{(k-j+1) !}\left(\begin{array}{c}
k-j+1 \\
i
\end{array}\right) \varepsilon^{i \xi^{k-j-i-1}} I_{k}\left(f_{k}\right) .
\end{aligned}
$$

The series defining $B_{1}^{\varepsilon}$ converges in $L^{2}(\Omega)$, because $F \in \Delta^{j, 2}$. As for $A_{2}^{\varepsilon, \xi}$, we have

$$
\left|B_{2}^{\varepsilon, \xi}\right| \leqslant \frac{1}{2} \frac{1}{\left(1-\varepsilon_{0}^{2}\right)^{1 / 2}} X_{j}
$$

with $X_{j}:=\left[\sum_{k=j+1}^{\infty}\{k ! /(k-j-1) !\}^{2}\left\{I_{k}\left(f_{k}\right)\right\}^{2}\right]^{1 / 2}$. This random variable is finite a.s., since $F \in \Delta^{j+1,2}$. Therefore

$$
\lim _{\xi \rightarrow 0} \frac{d_{j-1}^{\varepsilon+\xi}-d_{j-1}^{\varepsilon}}{\xi}=B_{1}^{\varepsilon}, \quad \text { a.s. }
$$

and the proof is complete.

Remark. For $j \in \mathbb{Z}^{+}$, the Sobolev spaces $\mathbb{D}^{j, 2}$ can be characterized as follows:

$$
\mathbb{D}^{j, 2}=\left\{F \in L^{2}(\Omega): \sum_{k=j}^{\infty}\left(\frac{k !}{(k-j) !}\right)^{2}(k-j) !\left\|f_{k}\right\|_{2}^{2}<\infty\right\} .
$$

Using the quotient criterion for comparison of series, one easily checks that $\mathbb{D}^{2 j, 2}=\Delta^{j, 2}$, $\forall j \in \mathbb{Z}^{+}$. Hence, the preceding proposition can be formulated in a more handy way, as follows.

Corollary 2.2. Let $F \in \bigcap_{j=0}^{\infty} \mathbb{D}^{j, 2}$. There exists a version of $\left\{F^{\varepsilon}, \varepsilon \in(0,1)\right\}$ which is $\mathscr{C}^{\infty}$ in $\varepsilon$ and

$$
\frac{\mathrm{d}^{j} F^{\varepsilon}}{\mathrm{d} \varepsilon^{j}}=\sum_{k=j}^{\infty} \frac{k !}{(k-j) !} \varepsilon^{k-j} I_{k}\left(f_{k}\right)
$$

$j \in \mathbb{Z}^{+}$, where the series in (2.3) converges in $L^{2}(\Omega)$.

In the proof of the main result of this section we deal with the random vector

$$
\hat{F}^{\varepsilon}=\frac{F^{\varepsilon}-\mathrm{E}(F)}{\varepsilon}, \quad 0<\varepsilon \leqslant 1 .
$$

Corollary 2.2 yields the following.

Corollary 2.3. Let $F \in \bigcap_{j=0}^{\infty} \mathbb{D}^{j, 2}$. There exists a version of $\left\{\hat{F}^{\varepsilon}, \varepsilon \in(0,1)\right\}$ which is $\mathscr{C}^{\infty}$ in $\varepsilon$ and 


$$
\begin{aligned}
& \frac{\mathrm{d}^{j} \hat{F}^{\varepsilon}}{\mathrm{d} \varepsilon^{j}}=\sum_{k=j+1}^{\infty} \frac{(k-1) !}{(k-(j+1)) !} \varepsilon^{k-(j+1)} I_{k}\left(f_{k}\right), \\
& \left.\frac{\mathrm{d}^{j} \hat{F}^{\varepsilon}}{\mathrm{d} \varepsilon^{j}}\right|_{\varepsilon=0}:=\lim _{\varepsilon \downarrow 0} \frac{\mathrm{d}^{j} \hat{F}^{\varepsilon}}{\mathrm{d} \varepsilon^{j}}=j ! I_{j+1}\left(f_{j+1}\right),
\end{aligned}
$$

$j \in \mathbb{Z}^{+}$. In particular, setting $\hat{F}^{0}=\lim _{\varepsilon \downarrow 0} \hat{F}^{\varepsilon}$, we have $\hat{F}^{0}=I_{1}\left(f_{1}\right)$.

Equation (2.4) can be checked by induction, using the same arguments as in the proof of Proposition 2.1.

Let $f: \mathbb{R}^{d} \rightarrow \mathbb{R}$ be a $\mathscr{C}^{\infty}$ function with compact support. Fix a multi-index $\alpha \in$ $\{1, \ldots, d\}^{k}, \alpha=\left(\alpha_{1}, \ldots, \alpha_{k}\right), k \geqslant 1$. The Leibniz formula yields, for $j \geqslant 1$,

$$
\frac{\mathrm{d}^{j}}{\mathrm{~d} \varepsilon^{j}}\left(f\left(\hat{F}^{\varepsilon}\right)\right)=\sum^{(j)}\left(\nabla_{\alpha}^{k} f\right)\left(\hat{F}^{\varepsilon}\right) \nabla^{\beta_{1}} \hat{F}^{\varepsilon, \alpha_{1}} \ldots \nabla^{\beta_{k}} \hat{F}^{\varepsilon, \alpha_{k}},
$$

with $\nabla_{\alpha}^{k}:=\partial^{k} / \partial x_{\alpha_{1}} \ldots \partial x_{\alpha_{k}}, \nabla^{\beta_{i}}:=d^{\beta_{i}} / d \varepsilon^{\beta_{i}}$, where the symbol $\sum^{(j)}$ is a shorthand for

$$
\sum_{k=1}^{j} \sum_{\substack{\beta_{1}+\ldots+\beta_{k}=j \\ \beta_{1}, \ldots, \beta_{k} \geqslant 1}} \sum_{\substack{\alpha \in\{1, \ldots, d\}^{k} \\ \alpha=\left(\alpha_{1}, \ldots, \alpha_{k}\right)}} c_{j}\left(\beta_{1}, \ldots, \beta_{k}\right)
$$

and the coefficients $c_{j}\left(\beta_{1}, \ldots, \beta_{k}\right)$ are obtained recursively, as follows:

$$
c_{j}\left(\beta_{1}, \ldots, \beta_{k}\right)=\sum_{i=1}^{k} c_{j-1}\left(\beta_{1}, \ldots, \beta_{i}-1, \ldots, \beta_{k}\right),
$$

with $c_{1}(1)=1$; for $\beta_{i}=1$ and $i<k, c_{j-1}\left(\beta_{1}, \ldots, \beta_{i}-1, \ldots, \beta_{k}\right)=0$; for $\beta_{k}=1$, $c_{j-1}\left(\beta_{1}, \ldots, \beta_{k}-1\right)=c_{j-1}\left(\beta_{1}, \ldots, \beta_{k-1}\right)$.

In the sequel we denote by $\Gamma_{G}$ the Malliavin matrix of a Wiener functional $G: \Omega \rightarrow \mathbb{R}^{d}$. Let $\Phi \in \mathbb{D}^{\infty}\left(\mathbb{R}^{d}\right)$ with $\operatorname{det} \Gamma_{\Phi}^{-1} \in \bigcap_{p \geqslant 1} L^{p}, \Psi \in \mathbb{D}^{\infty}$; for a multi-index $\alpha \in\{1, \ldots, d\}^{k}$, $\alpha=\left(\alpha_{1}, \ldots, \alpha_{k}\right), k \geqslant 1$, we define $H_{\alpha}(\Phi, \Psi)$ recursively, as follows:

$$
\begin{aligned}
H_{(i)}(\Phi, \Psi) & =\sum_{j=1}^{d} \delta\left(\Psi\left(\Gamma_{\Phi}^{-1}\right)^{i j} D \Phi^{j}\right), \\
H_{\alpha}(\Phi, \Psi) & =H_{\left(\alpha_{k}\right)}\left(\Phi, H_{\left(\alpha_{1}, \ldots, \alpha_{k-1}\right)}(\Phi, \Psi)\right),
\end{aligned}
$$

where $\delta$ denotes the Skorohod integral, which is the adjoint of the Malliavin derivative operator $D$.

This notation is appropriate to state the following version of the integration-by-parts formula:

$$
\mathrm{E}\left\{\left(\nabla_{\alpha}^{k} g\right)(\Phi) \Psi\right\}=\mathrm{E}\left\{g(\Phi) H_{\alpha}(\Phi, \Psi)\right\},
$$

where $g$ is any smooth function defined in $\mathbb{R}^{d}$.

A slight modification of Proposition 3.2.2 of Nualart (1998) yields the following estimate: for any $k \in \mathbb{N}, p \in[1, \infty)$ there exist $k^{\prime}, b, b^{\prime} \in(1, \infty), a, a^{\prime}, d, d^{\prime} \in \mathbb{N}$ such that 


$$
\left\|H_{\alpha}(\Phi, \Psi)\right\|_{k, p} \leqslant C(k, p, \alpha)\left(\left\|\Gamma_{\Phi}^{-1}\right\|_{k^{\prime}}\|\Phi\|_{d, b}^{a}\|\Psi\|_{d^{\prime}, b^{\prime}}^{a^{\prime}}\right),
$$

where $\|\cdot\|_{k, p}$ denotes the norm of the Sobolev spaces $\mathbb{D}^{k, p}$ in the Malliavin calculus.

Let $F \in \mathbb{D}^{\infty}\left(\mathbb{R}^{d}\right)$. The family of $\mathbb{R}^{d}$-valued random vectors $\left\{F^{\varepsilon}, \varepsilon \in(0,1]\right\}$ defined by (2.1) is said to be uniformly non-degenerate if the next condition is satisfied:

$$
\left\|\Gamma_{F^{\varepsilon}}^{-1}\right\|_{p} \leqslant C \varepsilon^{-2}
$$

for any $\varepsilon \in(0,1], p \in[1, \infty)$.

Remark. Consider the Ornstein-Uhlenbeck semigroup $\left\{T_{t}, t \geqslant 0\right\}$, which is a contraction operator with respect to any Sobolev norm $\|\cdot\|_{k, p}$ (see, for example, Nualart (1998)). Then $T_{t} F=\sum_{n=0}^{\infty} \mathrm{e}^{-n t} I_{n}\left(f_{n}\right)$ and therefore $F^{\varepsilon}=T_{-\log \varepsilon} F$. It follows that $\left\{F^{\varepsilon}, \varepsilon \in(0,1]\right\} \subset$ $\mathbb{D}^{\infty}\left(\mathbb{R}^{d}\right)$.

Remark. Set $\sigma^{2}=\operatorname{det}\left[\operatorname{cov}\left\{I_{1}\left(f_{1}\right)\right\}\right]$. The uniform non-degeneracy condition (2.10) implies that $\sigma^{2}>0$.

Indeed, let

$$
M_{r}^{\varepsilon}=\sum_{n=1}^{\infty} \varepsilon^{n-1} n I_{n-1}\left(f_{n}(\cdot, r)\right), \quad r \in T,
$$

and denote by $H^{\varepsilon}$ the matrix $\left(\left\langle M^{\varepsilon, i}, M^{\varepsilon, j}\right\rangle_{L^{2}(T)}\right)_{1 \leqslant i, j \leqslant d}$. Then $D_{r} F^{\varepsilon}=\varepsilon M_{r}^{\varepsilon}$ and (2.10) is equivalent to

$$
\sup _{\varepsilon \in(0,1]}\left\|\left(H^{\varepsilon}\right)^{-1}\right\|_{p} \leqslant C, \quad \forall p \in[1, \infty)
$$

Next we prove that

$$
L^{1}-\lim _{\varepsilon \downarrow 0} H^{\varepsilon}=\operatorname{cov}\left\{I_{1}\left(f_{1}\right)\right\} .
$$

Then, the Fatou lemma and (2.11) yield $\sigma^{2}>0$. For simplicity we suppose that $d=1$. Then

$$
\mathrm{E}\left|H^{\varepsilon}-\operatorname{var}\left\{I_{1}\left(f_{1}\right)\right\}\right| \leqslant T_{1}^{\varepsilon}+T_{2}^{\varepsilon},
$$

with

$$
\begin{aligned}
& T_{1}^{\varepsilon}=\mathrm{E}\left|\sum_{n=2}^{\infty} \varepsilon^{2(n-1)} n^{2} \int_{T}\left\{I_{n-1}\left(f_{n}(\cdot, r)\right)\right\}^{2} \mathrm{~d} r\right| \\
& T_{2}^{\varepsilon}=\mathrm{E}\left|\int_{T} \sum_{\substack{n, m=1 \\
n \neq m}}^{\infty} \varepsilon^{n+m-2} n m I_{n-1}\left(f_{n}(\cdot, r)\right) I_{m-1}\left(f_{m}(\cdot, r)\right) \mathrm{d} r\right| .
\end{aligned}
$$

We have

$$
T_{1}^{\varepsilon} \leqslant \varepsilon^{2} \sum_{n=2}^{\infty} n^{2}(n-1) !\left\|f_{n}\right\|_{2}^{2} .
$$


Analogously, $T_{2}^{\varepsilon}=\varepsilon T_{21}^{\varepsilon}$, with

$$
T_{21}^{\varepsilon}=\mathrm{E}\left|\int_{T} \sum_{\substack{n, m=1 \\ n \neq m}}^{\infty} \varepsilon^{n+m-3} n m I_{n-1}\left(f_{n}(\cdot, r)\right) I_{m-1}\left(f_{m}(\cdot, r)\right) \mathrm{d} r\right| .
$$

Fix $\alpha>1$. The Schwarz inequality implies that

$$
\begin{aligned}
T_{21}^{\varepsilon} & \leqslant \mathrm{E} \mid \sum_{\substack{n, m=1 \\
n \neq m}}^{\infty} \varepsilon^{n+m-3} n m\left(\int_{T}\left\{I_{n-1}\left(f_{n}(\cdot, r)\right)\right\}^{2} \mathrm{~d}_{r}\right)^{1 / 2}\left(\int_{T}\left\{I_{m-1}\left(f_{m}(\cdot, r)\right)\right\}^{2} \mathrm{~d} r\right)^{1 / 2} \\
& \leqslant \mathrm{E}\left\{\sum_{n=1}^{\infty} n\left(\int_{T}\left\{I_{n-1}\left(f_{n}(\cdot, r)\right)\right\}^{2} \mathrm{~d} r\right)^{1 / 2}\right\}^{2} \\
& \leqslant C \sum_{n=1}^{\infty} n^{2+\alpha}(n-1) !\left\|f_{n}\right\|_{2}^{2} .
\end{aligned}
$$

Since $F \in \mathbb{D}^{\infty}$, the series $\sum_{n=1}^{\infty} n^{2+\alpha}(n-1) !\left\|f_{n}\right\|_{2}^{2}$ converges. Therefore

$$
\lim _{\varepsilon \downarrow 0}\left(T_{1}^{\varepsilon}+T_{2}^{\varepsilon}\right)=0 \text {, }
$$

proving (2.12).

We now give the main result of this section.

Theorem 2.4. Let $\left\{F^{\varepsilon}, \varepsilon \in(0,1]\right\}$ be uniformly non-degenerate. The density $p^{\varepsilon}(y)$, for $y=\mathrm{E}\left(F^{\varepsilon}\right)=\mathrm{E}(F)$, has the Taylor expansion

$$
p^{\varepsilon}(y)=\frac{1}{\varepsilon^{d}}\left(\frac{1}{(2 \pi)^{d / 2} \sigma}+\sum_{j=1}^{N} \varepsilon^{j} \frac{1}{j !} p_{j}+\varepsilon^{N+1} \tilde{p}_{N+1}^{\varepsilon}\right) .
$$

The coefficients $p_{j}$ are null for odd $j$. For even $j \in\{1,2, \ldots, N\}$,

$$
p_{j}=\mathrm{E}\left(1_{\left\{I_{1}\left(f_{1}\right)>0\right\}} P_{j}\right),
$$

with $P_{j}$ belonging to $\oplus_{k=0}^{3 j+d} \mathscr{H}_{k}$, $\mathscr{H}_{k}$ being the kth Wiener chaos, and

$$
P_{j}=\sum^{(j)} H_{(1, \ldots, d)}\left(I_{1}\left(f_{1}\right), H_{\alpha}\left(I_{1}\left(f_{1}\right), \prod_{\ell=1}^{k} \beta_{\ell} ! I_{\beta_{\ell+1}}\left(f_{\beta_{\ell}+1}^{\alpha_{\ell}}\right)\right)\right) .
$$

In addition, if for any $j \in \mathbb{Z}^{+}, k \in \mathbb{N}, p \in[1, \infty)$,

$$
\sup _{\varepsilon \in(0,1]}\left\|\frac{\mathrm{d}^{j}}{\mathrm{~d}^{j}} \hat{F}^{\varepsilon}\right\|_{k, p} \leqslant C,
$$

then $\sup _{\varepsilon \in(0,1]}\left|\tilde{p}_{N+1}^{\varepsilon}\right|$ is finite. 


\section{Remarks}

(1) The identities (2.14) and (2.15) express the fact that $p_{j}, j=1, \ldots, N$, are the densities at $x=0$ of the Radon measures defined by

$$
g \mapsto \mathrm{E}\left\{g\left(I_{1}\left(f_{1}\right)\right) \sum^{(j)} H_{\alpha}\left(I_{1}\left(f_{1}\right), \prod_{\ell=1}^{k} \beta_{\ell} ! I_{\beta_{\ell}+1}\left(f_{\beta_{\ell}+1}^{\alpha_{p}}\right)\right)\right\},
$$

for any smooth $g$ (see, for instance, Corollary 3.2.1 of Nualart (1998)).

(2) As will become clear from the proof of Theorem $2.4, \tilde{p}_{N+1}^{\varepsilon}$ is also the density of a Radon measure depending on $\varepsilon$. The last assertion of the theorem gives a sufficient condition ensuring the uniform boundedness of this density. In this case, the last term in the expansion (2.13) is $O\left(\varepsilon^{N+1}\right)$ as $\varepsilon \downarrow 0$.

Proof of Theorem 2.4. Let $\hat{p}^{\varepsilon}$ denote the density of $\hat{F}^{\varepsilon}=\left\{F^{\varepsilon}-E(F)\right\} / \varepsilon$. Clearly $p^{\varepsilon}(y)=\left(1 / \varepsilon^{d}\right) \hat{p}^{\varepsilon}(0)$. Therefore we shall find an expansion for $\hat{p}^{\varepsilon}(0)$. Let $f: \mathbb{R}^{d} \rightarrow \mathbb{R}$ be a $\mathscr{C}^{\infty}$ function with bounded support. The mapping $\varepsilon \mapsto f\left(\hat{F}^{\varepsilon}\right)$ is $\mathscr{C}^{\infty}$, a.s., therefore

$$
f\left(\hat{F}^{\varepsilon}\right)=f\left(\hat{F}^{0}\right)+\left.\sum_{j=1}^{N} \frac{1}{j !} \varepsilon^{j} \frac{\mathrm{d}^{j}}{\mathrm{~d} \varepsilon^{j}}\left\{f\left(\hat{F}^{\varepsilon}\right)\right\}\right|_{\varepsilon=0}+\left.\varepsilon^{N+1} \int_{0}^{1} \frac{(1-t)^{N}}{N !} \frac{\mathrm{d}^{N+1}}{\mathrm{~d} \eta^{N+1}}\left\{f\left(\hat{F}^{\eta}\right)\right\}\right|_{\eta=t \varepsilon} \mathrm{d} t .
$$

Next, we take expectations of both sides of the preceding equality; we use (2.6), (2.5) and the integration-by-parts formula (2.8) to obtain

$$
\begin{aligned}
\mathrm{E}\left\{f\left(\hat{F}^{\varepsilon}\right)\right\}= & \mathrm{E}\left\{f\left(I_{1}\left(f_{1}\right)\right)\right\}+\sum_{j=1}^{N} \frac{1}{j !} \varepsilon^{j} \mathrm{E}\left\{f\left(I_{1}\left(f_{1}\right)\right) \sum^{(j)} H_{\alpha}\left(I_{1}\left(f_{1}\right), \prod_{\ell=1}^{k} \beta_{\ell} ! I_{\beta_{\ell}+1}\left(f_{\beta_{\ell}+1}^{\alpha_{\ell}}\right)\right)\right\} \\
& +\varepsilon^{N+1} \int_{0}^{1} \frac{(1-t)^{N}}{N !} \mathrm{E}\left\{f\left(\hat{F}^{\varepsilon t}\right) \sum^{(N+1)} H_{\alpha}\left(\hat{F}^{\varepsilon t},\left.\prod_{\ell=1}^{k} \frac{d^{\beta_{\ell}} \hat{F}^{\eta, \alpha_{\ell}}}{d \eta^{\beta_{\ell}}}\right|_{\eta=\varepsilon t}\right)\right\} \mathrm{d} t .
\end{aligned}
$$

The assumptions of the theorem ensure (see Remarks directly after (2.10)) that the Radon measures defined by $\mathrm{E}\left\{f\left(\hat{F}^{\varepsilon}\right)\right\}, \mathrm{E}\left\{f\left(I_{1}\left(f_{1}\right)\right)\right\}, \mathrm{E}\left\{f\left(I_{1}\left(f_{1}\right)\right) Q^{j}\right\}, \quad j=1, \ldots, N$, $\mathrm{E}\left\{f\left(\hat{F}^{\varepsilon t}\right) Q^{N+1, \varepsilon}\right\}$, with

$$
\begin{aligned}
Q^{j} & =\sum^{(j)} H_{\alpha}\left(I_{1}\left(f_{1}\right), \prod_{\ell=1}^{k} \beta_{\ell} ! I_{\beta_{\ell}+1}\left(f_{\beta_{\ell}+1}^{\alpha_{\ell}}\right)\right), \\
Q^{N+1, \varepsilon} & =\sum^{(N+1)} H_{\alpha}\left(\hat{F}^{\varepsilon t},\left.\prod_{\ell=1}^{k} \frac{\mathrm{d}^{\beta_{\ell}}}{\mathrm{d} \eta^{\beta_{\ell}}} \hat{F}^{\eta, \alpha_{\ell}}\right|_{\eta=\varepsilon t}\right),
\end{aligned}
$$

possess $\mathscr{C}^{\infty}$ densities. Moreover, a new integration-by-parts in (2.17) yields (see Corollary 3.2.1 of Nualart (1998)) 


$$
\hat{p}^{\varepsilon}(0)=\frac{1}{(2 \pi)^{d / 2} \sigma}+\sum_{j=1}^{N} \frac{1}{j !} \varepsilon^{j} \mathrm{E}\left(1_{\left\{I_{1}\left(f_{1}\right)>0\right\}} P_{j}\right)+\varepsilon^{N+1} \tilde{p}_{N+1}^{\varepsilon},
$$

with

$$
\tilde{p}_{N+1}^{\varepsilon}=\int_{0}^{1} \frac{(1-t)^{N}}{N !} \mathrm{E}\left\{1_{\left\{\hat{F}^{\varepsilon t}>0\right\}} H_{(1, \ldots, d)}\left(\hat{F}^{\varepsilon t}, \sum^{(N+1)} H_{\alpha}\left(\hat{F}^{\varepsilon t},\left.\prod_{\ell=1}^{k} \frac{\mathrm{d}^{\beta_{\ell}}}{\mathrm{d} \eta^{\beta_{\ell}}} \hat{F}^{\eta, \alpha_{\ell}}\right|_{\eta=\varepsilon t}\right)\right)\right\} \mathrm{d} t .
$$

Consider the Wiener-chaos decomposition

$$
\hat{F}^{\varepsilon}=\sum_{n=1}^{\infty} \varepsilon^{n-1} I_{n}\left(f_{n}\right)
$$

and an even smooth function $f$. The Wiener measure is invariant under the transformation $\mathscr{Z}(\omega)=-\omega$. Thus, $f\left(\hat{F}^{-\varepsilon}\right)$ and $f\left(\hat{F}^{\varepsilon}\right)$ have the same law and the odd coefficients in the Taylor expansion are zero.

The fact that $P_{j}$ has a finite Wiener-chaos decomposition, more precisely, $P_{j} \in$ $\mathscr{T}_{3 j+d}=\oplus_{k=0}^{3 j+d} \mathscr{H}_{k}$, follows from Lemma 2.5. Indeed, for any $k \in\{1, \ldots, j\}, \Psi:=$ $\prod_{\ell=1}^{k} \beta_{\ell} ! I_{\beta_{\ell}+1}\left(f_{\beta_{\ell}+1}^{\alpha_{\ell}}\right) \in \mathscr{T}_{2 j}$, since $\beta_{1}+\ldots+\beta_{k}=j$. Consequently $Q_{j} \in \mathscr{T}_{3 j}$, because the length of $\alpha$ is $k$. Finally, since $P_{j}=H_{(1, \ldots, d)}\left(I_{1}\left(f_{1}\right), Q_{j}\right)$, Lemma 2.5 yields $P_{j} \in \mathscr{T}_{3 j+d}$.

We now want to give a uniform bound for $\tilde{p}_{N+1}^{\varepsilon}$ (see (2.19)). Set $G^{\varepsilon}=$ $\prod_{\ell=1}^{k}\left(\mathrm{~d}^{\beta_{\curlywedge}} / \mathrm{d} \varepsilon^{\beta_{\succ}}\right) \hat{F}^{\varepsilon}$. Clearly, it suffices to show that

$$
\sup _{0<\varepsilon \leqslant 1} \mathrm{E}\left\{\left|H_{(1, \ldots, d)}\left(\hat{F}^{\varepsilon}, H_{\alpha}\left(\hat{F}^{\varepsilon}, G^{\varepsilon}\right)\right)\right|\right\} \leqslant C,
$$

for any $\alpha \in\{1, \ldots, d\}^{k}, \beta_{1}+\ldots+\beta_{k}=N+1, k=1, \ldots, N+1$, and some finite $C>0$. The estimate (2.9) yields, for some $k, b, b^{\prime} \in(1, \infty)$ and $a, a^{\prime}, d, d^{\prime} \in \mathbb{N}$,

$$
\mathrm{E}\left|H_{(1, \ldots, d)}\left(\hat{F}^{\varepsilon}, H_{\alpha}\left(\hat{F}^{\varepsilon}, G^{\varepsilon}\right)\right)\right| \leqslant C\left(\left\|\Gamma_{\hat{F}^{\varepsilon}}^{-1}\right\|_{k}\left\|\hat{F}^{\varepsilon}\right\|_{d, b}^{a}\left\|G^{\varepsilon}\right\|_{d^{\prime}, b^{\prime}}^{a^{\prime}}\right) .
$$

Therefore, the non-degeneracy condition $\left\|\Gamma_{F^{\varepsilon}}^{-1}\right\|_{p} \leqslant C \varepsilon^{-2}, \forall p \in(1, \infty)$ together with condition (2.16) yields (2.20). This finishes the proof of the theorem.

Lemma 2.5. Let $\Phi$ be a non-degenerate d-dimensional random vector belonging to the first chaos $\mathscr{H}_{1}, \Psi \in \mathscr{T}_{\ell}, \ell \geqslant 0$. For any multi-index $\alpha=\left(\alpha_{1}, \ldots, \alpha_{r}\right) \in\{1, \ldots, d\}^{r}$, the random variable $H_{\alpha}(\Phi, \Psi)$ belongs to $\mathscr{T} \ell+r$.

Proof. We shall proceed by induction on the length of $\alpha$. Set $\left(b^{i, j}\right)_{i, j=1, \ldots, d}=(\operatorname{cov} \Phi)^{-1}$ and $\Phi=I_{1}(f)$. Then, for any $i \in\{1, \ldots, d\}$,

$$
H_{(i)}(\Phi, \Psi)=\sum_{j=1}^{d} b^{i j} \delta(\Psi f) \in \mathscr{T}_{\ell+1} .
$$

Assume that the statement holds for any multi-index of length $r-1$. Let $\alpha=$ $\left(\alpha_{1}, \ldots, \alpha_{r}\right) \in\{1, \ldots, d\}^{r}$. By $(2.6)$, 


$$
H_{\alpha}(\Phi, \Psi)=H_{\left(\alpha_{r}\right)}(\Phi, \tilde{\Psi})=\sum_{j=1}^{d} b^{\alpha_{r}, j} \delta(\tilde{\Psi} f),
$$

with $\tilde{\Psi} \in \mathscr{T}_{\ell+r-1}$. Thus $H_{\alpha}(\Phi, \Psi) \in \mathscr{T}_{\ell+r}$ and the proof is complete.

Remark. Let $\Phi(h)=\mathrm{E}(F)+\sum_{n=1}^{\infty} \int_{T} n f_{n}\left(s_{1}, \ldots, s_{n}\right) \mathrm{d} h_{s_{1}} \ldots \mathrm{d} h_{s_{n}}, h \in \mathscr{H}$. Note that the series defining $\Phi(h)$ is absolutely convergent, owing to the condition $\sum_{n=1}^{\infty} n !\left\|f_{n}\right\|_{2}^{2}<+\infty$. Assume that there exists a sequence $\left\{\omega^{n}, n \in \mathbb{N}\right\} \subset \mathscr{H}$ such that $P-\lim _{n \rightarrow \infty} \Phi\left(\omega^{n}\right)=F$ and, moreover, for any $h \in \mathscr{H}, n \in \mathbb{N}$, there exists an absolutely continuous transformation $T_{n}^{h}: \Omega \rightarrow \Omega$ such that $P-\lim _{n \rightarrow \infty} F \circ T_{n}^{h}=\Phi(h)$. If, in addition $F^{\varepsilon} \in \mathbb{D}^{\infty}$ and $\left\|\operatorname{det} \Gamma_{F^{\varepsilon}}^{-1}\right\|_{p}<+\infty, \forall p \in(1, \infty)$, Theorem 3.41 of Aida et al. (1993) establishes the following characterization for the points of positive density for $F^{\varepsilon}$ :

$$
\left\{p^{\varepsilon}(y)>0\right\}=\{y: \exists h \in \mathscr{H}: \Phi(h)=y \quad \text { and } \quad D \Phi(h) \text { surjective }\} .
$$

Assume that the family $\left\{F^{\varepsilon}, \varepsilon \in(0,1]\right\}$ possesses the approximating property described before and is uniformly non-degenerate. Then, for $y=\mathrm{E}(F), p^{\varepsilon}(y)>0$. Indeed, $\Phi(0)=\mathrm{E}(F)=y$ and, for any $k \in \mathscr{H}$,

$$
D \Phi(0)(k)=\int_{T} f_{1}(s) k(s) \mathrm{d} \mu(s) .
$$

Thus, since $\sigma^{2}:=\operatorname{det}\left[\operatorname{cov}\left\{I_{1}\left(f_{1}\right)\right\}\right]>0, D \Phi(h)$ is surjective.

\section{Applications}

We devote this section to study two examples where Theorem 2.4 can be applied.

\subsection{A hyperbolic stochastic partial differential equation}

Let $T=[0,1]^{2}$ and $\left\{W_{s, t},(s, t) \in T\right\}$ be a Wiener sheet. Consider the hyperbolic stochastic partial differential equation

$$
\frac{\partial^{2} X_{s, t}}{\partial s \partial t}=a_{3}\left(X_{s, t}, s, t\right) \dot{W}_{s, t}+a_{4}\left(X_{s, t}, s, t\right)+a_{1}(s, t) \frac{\partial X_{s, t}}{\partial s}+a_{2}(s, t) \frac{\partial X_{s, t}}{\partial t},
$$

with deterministic initial condition $X_{s, t}=x_{0}$ if $(s, t) \in T, s \cdot t=0$. We refer to Farré and Nualart (1993), and especially to Rovira and Sanz-Solé (1996) for results on this equation used in this section.

Here we shall deal with the particular situation made precise in the following set of assumptions.

(H1) $a_{i}: T \rightarrow \mathbb{R}, \quad i=1,2$ are differentiable and bounded with bounded first partial derivatives.

(H2) $a_{i}: \mathbb{R} \times T \rightarrow \mathbb{R}, i=3,4$ are linear in the space variable, which means that

$$
a_{i}(x, s, t)=a_{i 1}(s, t) x+a_{i 2}(s, t) .
$$


In addition, $a_{31}, a_{32}, a_{41}$ and $a_{42}$ are supposed to be continuous.

A solution of (3.1) is a stochastic process $\left\{X_{s, t},(s, t) \in T\right\}$ satisfying

$$
X_{s, t}=x_{0}+\int_{R_{s, t}} \gamma_{s, t}(u, v)\left\{a_{3}\left(X_{u, v}, u, v\right) \mathrm{d} W_{u, v}+a_{4}\left(X_{u, v}, u, v\right) \mathrm{d} u \mathrm{~d} v\right\},
$$

where $R_{s, t}$ denotes the rectangle $[0, s] \times[0, t]$ and $\gamma_{s, t}(u, v)$ is the Green function associated with the second-order differential operator

$$
\mathscr{C} f(s, t)=\frac{\partial^{2} f(s, t)}{\partial s \partial t}-a_{1}(s, t) \frac{\partial f(s, t)}{\partial t}-a_{2}(s, t) \frac{\partial f(s, t)}{\partial s} .
$$

Here are some properties of the Green function; their proofs are given in Rovira and SanzSolé (1996):

boundedness:

$$
\sup _{(s, t) \in T(u, v) \leqslant(s, t)} \sup _{s, t} \mid \gamma_{s, v) \mid \leqslant C}
$$

Lipschitz property:

$$
\begin{gathered}
\sup _{(s, t) \in T}\left|\gamma_{s, t}(\bar{u}, \bar{v})-\gamma_{s, t}(u, v)\right| \leqslant C\{|\bar{u}-u|+|\bar{v}-v|\},(\bar{u}, \bar{v}),(u, v) \leqslant(s, t), \\
\sup _{(u, v) \in T}\left|\gamma_{\bar{s}, \bar{t}}(u, v)-\gamma_{s, t}(u, v)\right| \leqslant C\{|\bar{s}-s|+|\bar{t}-t|\},(\bar{s}, \bar{t}),(s, t) \geqslant(u, v) ;
\end{gathered}
$$

positivity:

$$
\begin{array}{ll}
\gamma_{s, t}(s, v)=\exp \left(\int_{v}^{t} a_{2}(s, w) \mathrm{d} w\right), & 0 \leqslant v \leqslant t, \\
\gamma_{s, t}(u, t)=\exp \left(\int_{u}^{s} a_{1}(r, t) \mathrm{d} r\right), & 0 \leqslant u \leqslant s .
\end{array}
$$

Theorem 2.1 of Rovira and Sanz-Solé (1996) proves the existence and uniqueness of a continuous and adapted process $\left\{X_{s, t},(s, t) \in T\right\}$ bounded in $L^{p}$, for any $p \geqslant 2$. Moreover, $X_{s, t} \in \mathbb{D}^{\infty}, \forall(s, t) \in T$. For any $\varepsilon \in(0,1]$ set

$$
X_{s, t}^{\varepsilon}=x_{0}+\int_{R_{s, t}} \gamma_{s, t}(u, v)\left\{\varepsilon a_{3}\left(X_{u, v}^{\varepsilon}, u, v\right) \mathrm{d} W_{u, v}+a_{4}\left(X_{u, v}^{\varepsilon}, u, v\right) \mathrm{d} u \mathrm{~d} v\right\}
$$

and, for any $h \in \mathscr{H}$, the Cameron-Martin space associated with $\left\{W_{s, t},(s, t) \in T\right\}$,

$$
S_{s, t}^{h}=x_{0}+\int_{R_{s, t}} \gamma_{s, t}(u, v)\left\{a_{3}\left(S_{u, v}^{h}, u, v\right) \mathrm{d} h_{u, v}+a_{4}\left(S_{u, v}^{h}, u, v\right) \mathrm{d} u \mathrm{~d} v\right\} .
$$

Proposition 3.1. Assume (H1) and (H2). For any $z \in T, z=(s, t)$, st $\neq 0$, let

$$
X_{z}=\mathrm{E} X_{z}+\sum_{n=1}^{\infty} I_{n}\left(f_{n}\right)
$$


be the Wiener-chaos decomposition of the solution of (3.2) at $z=(s, t)$. Then, for any $\varepsilon \in(0,1]$,

$$
X_{z}^{\varepsilon}=\mathrm{E} X_{z}+\sum_{n=1}^{\infty} \varepsilon^{n} I_{n}\left(f_{n}\right)
$$

Proof. By a result proved by Stroock (1987), $f_{n}(\alpha)=(1 / n !) \mathrm{E}\left(D_{\alpha}^{n} X_{z}\right), \alpha=\left(\alpha_{1}, \ldots, \alpha_{n}\right)$. Thus, if $X_{z}^{\varepsilon}=\mathrm{E} X_{z}^{\varepsilon}+\sum_{n=1}^{\infty} I_{n}\left(f_{n}^{\varepsilon}\right)$, it suffices to prove $\mathrm{E} X_{z}=\mathrm{E} X_{z}^{\varepsilon}$ and $\mathrm{E}\left(D_{\alpha}^{n} X_{z}^{\varepsilon}\right)=$ $\varepsilon^{n} \mathrm{E}\left(D_{\alpha}^{n} X_{z}\right), n \geqslant 1$.

Taking expectations in (3.2) and (3.3) by uniqueness of solution we immediately obtain

$$
\mathrm{E} X_{z}=\mathrm{E} X_{z}^{\varepsilon}=S_{z}^{0}
$$

Fix $N \in \mathbb{N}, \quad \alpha_{1}, \ldots, \alpha_{N} \in R_{z}$. Denote by $\alpha$ the vector $\left(\alpha_{1}, \ldots, \alpha_{N}\right)$; set $\alpha^{i}=$ $\left(\alpha_{1}, \ldots, \alpha_{i-1}, \alpha_{i+1}, \ldots, \alpha_{N}\right), N \geqslant 2, \sup \boldsymbol{\alpha}=\alpha_{1} \vee \ldots \vee \alpha_{N}$. The particular form of the coefficients $a_{i}, i=3,4$ and the rules of Malliavin calculus yield the following expressions for $N \geqslant 2$ :

$$
\begin{aligned}
D_{\boldsymbol{\alpha}}^{N} X_{z}= & \sum_{i=1}^{N} a_{3,1}\left(\alpha_{i}\right) \gamma_{z}\left(\alpha_{i}\right) D_{\alpha^{i}}^{N-1} X_{\alpha_{i}} \\
& +\int_{[\sup \boldsymbol{\alpha}, z]} \gamma_{z}(\eta)\left\{a_{3,1}(\eta) D_{\boldsymbol{\alpha}}^{N} X_{\eta} \mathrm{d} W_{\eta}+a_{4,1}(\eta) D_{\boldsymbol{\alpha}}^{N} X_{\eta} \mathrm{d} \eta\right\} \\
D_{\boldsymbol{\alpha}}^{N} X_{z}^{\varepsilon}= & \sum_{i=1}^{N} \varepsilon a_{3,1}\left(\alpha_{i}\right) \gamma_{z}\left(\alpha_{i}\right) D_{\alpha^{i}}^{N-1} X_{\alpha_{i}}^{\varepsilon} \\
& +\int_{[\sup \boldsymbol{\alpha}, z]} \gamma_{z}(\eta)\left\{\varepsilon a_{3,1}(\eta) D_{\boldsymbol{\alpha}}^{N} X_{\eta}^{\varepsilon} \mathrm{d} W_{\eta}+a_{4,1}(\eta) D_{\boldsymbol{\alpha}}^{N} X_{\eta}^{\varepsilon} \mathrm{d} \eta\right\}
\end{aligned}
$$

Let $U_{\boldsymbol{a}}^{N}(z), N \geqslant 1$, be the solution of the equation

$$
U_{\boldsymbol{\alpha}}^{N}(z)=1+\int_{[\sup \boldsymbol{\alpha}, z]} \gamma_{z}(\eta) a_{4,1}(\eta) U_{\boldsymbol{\alpha}}^{N}(\eta) \mathrm{d} \eta
$$

Then, clearly

$$
\begin{aligned}
& \mathrm{E}\left(D_{\boldsymbol{\alpha}}^{N} X_{z}\right)=\left(\sum_{i=1}^{N} a_{3,1}\left(\alpha_{i}\right) \gamma_{z}\left(\alpha_{i}\right) \mathrm{E}\left(D_{\alpha^{i}}^{N-1} X_{\alpha_{i}}\right)\right) U_{\boldsymbol{\alpha}}^{N}(z), \\
& \mathrm{E}\left(D_{\boldsymbol{\alpha}}^{N} X_{z}^{\varepsilon}\right)=\left(\sum_{i=1}^{N} \varepsilon a_{3,1}\left(\alpha_{i}\right) \gamma_{z}\left(\alpha_{i}\right) \mathrm{E}\left(D_{\alpha^{i}}^{N-1} X_{\alpha_{i}}^{\varepsilon}\right)\right) U_{\boldsymbol{\alpha}}^{N}(z) .
\end{aligned}
$$


For $N=1$,

$$
\begin{aligned}
& \mathrm{E}\left(D_{\alpha} X_{z}\right)=\gamma_{z}(\alpha)\left\{a_{3,1}(\alpha) \mathrm{E} X_{\alpha}+a_{3,2}(\alpha)\right\} U_{\alpha}^{1}(z), \\
& \mathrm{E}\left(D_{\alpha} X_{z}^{\varepsilon}\right)=\varepsilon \gamma_{z}(\alpha)\left\{a_{3,1}(\alpha) \mathrm{E} X_{\alpha}^{\varepsilon}+a_{3,2}(\alpha)\right\} U_{\alpha}^{1}(z) .
\end{aligned}
$$

Thus, $\mathrm{E}\left(D_{\alpha} X_{z}^{\varepsilon}\right)=\varepsilon \mathrm{E}\left(D_{\alpha} X_{z}\right)$, because $\mathrm{E} X_{\alpha}=\mathrm{E} X_{\alpha}^{\varepsilon}$. This fact and (3.4) allow us to finish the proof using a recursive argument.

In the sequel we fix $z \in T$ not belonging to the axes. The following notation will be used. Set

$$
\hat{X}_{z}^{\varepsilon}=\frac{X_{z}^{\varepsilon}-S_{z}^{0}}{\varepsilon}, \quad X_{j}^{\varepsilon}(z)=\frac{\mathrm{d}^{j}}{\mathrm{~d} \varepsilon^{j}} X_{z}^{\varepsilon}, \quad \hat{X}_{j}^{\varepsilon}(z)=\frac{\mathrm{d}^{j}}{\mathrm{~d} \varepsilon^{j}} \hat{X}_{z}^{\varepsilon}, \quad j \in \mathbb{N} .
$$

By Corollary 2.2 applied to $F=X_{z}$ we know that these derivatives exist. One easily checks that

$$
\begin{aligned}
& X_{1}^{\varepsilon}(z)=\int_{R_{z}} \gamma_{z}(\eta)\left[\left\{a_{3,1}(\eta) X_{\eta}^{\varepsilon}+a_{3,2}(\eta)\right\} \mathrm{d} W_{\eta}+\varepsilon a_{3,1}(\eta) X_{1}^{\varepsilon}(\eta) \mathrm{d} W_{\eta}+a_{4,1}(\eta) X_{1}^{\varepsilon}(\eta) \mathrm{d} \eta\right], \\
& X_{j}^{\varepsilon}(z)=\int_{R_{z}} \gamma_{z}(\eta)\left\{j a_{3,1}(\eta) X_{j-1}^{\varepsilon}(\eta) \mathrm{d} W_{\eta}+\varepsilon a_{3,1}(\eta) X_{j}^{\varepsilon}(\eta) \mathrm{d} W_{\eta}+a_{4,1}(\eta) X_{j}^{\varepsilon}(\eta) \mathrm{d} \eta\right\}, \quad j \geqslant 2 .
\end{aligned}
$$

Let $X_{j}^{0}(z)=\lim _{\varepsilon \downarrow 0} X_{j}^{\varepsilon}(z), j \geqslant 1$. Then $X_{j}^{0}(z)$ satisfies the following stochastic differential equations:

$$
\begin{gathered}
X_{1}^{0}(z)=\int_{R_{z}} \gamma_{z}(\eta)\left[\left\{a_{3,1}(\eta) S_{\eta}^{0}+a_{3,2}(\eta)\right\} \mathrm{d} W_{\eta}+a_{4,1}(\eta) X_{1}^{0}(\eta) \mathrm{d} \eta\right], \\
X_{j}^{0}(z)=\int_{R_{z}} \gamma_{z}(\eta)\left\{j a_{3,1}(\eta) X_{j-1}^{0}(\eta) \mathrm{d} W_{\eta}+a_{4,1}(\eta) X_{j}^{0}(\eta) \mathrm{d} \eta\right\} .
\end{gathered}
$$

Lemma 3.2. We assume $(\mathrm{H} 1)$ and $(\mathrm{H} 2)$. Then

$$
\hat{X}_{j}^{\varepsilon}(z)=\frac{1}{j+1}\left(X_{j+1}^{0}(z)+\varepsilon \int_{0}^{1}\left(1-\xi^{j+1}\right) X_{j+2}^{\xi \varepsilon}(z) \mathrm{d} \xi\right),
$$

$j \in \mathbb{Z}^{+}$, where, by convention, $\hat{X}_{0}^{\varepsilon}(z)=\hat{X}_{z}^{\varepsilon}$.

Proof. For $j=0$ the identity (3.9) follows from a Taylor expansion of $X_{z}^{\varepsilon}$ around $\varepsilon=0$, taking into account that $X_{z}^{0}=S_{z}^{0}$. An easy recursive argument establishes (3.9) for any $j \geqslant 1$.

In the next proposition we check assumption (2.16) of Theorem 2.4 for $\hat{F}^{\varepsilon}=\hat{X}_{z}^{\varepsilon}$. 
Remark. We know that there exists a version of $\left\{\hat{X}_{j}^{\varepsilon}(z), z \in T\right\}$ which is continuous in $\varepsilon$. From the previous lemma and (2.5) we obtain

$$
I_{n}\left(f_{n}\right)=\frac{X_{n}^{0}(z)}{n !}, \quad n \geqslant 1 .
$$

Proposition 3.3. Suppose that (H1) and (H2) are satisfied. For any $j \in \mathbb{Z}^{+}, k \in \mathbb{N}$, $p \in(1, \infty)$,

$$
\sup _{0<\varepsilon \leqslant 1}\left\|\frac{\mathrm{d}^{j}}{\mathrm{~d} \varepsilon^{j}} \hat{X}_{z}^{\varepsilon}\right\|_{k, p} \leqslant C .
$$

Proof. Because of (3.9) the proof follows from the following facts:

$$
\begin{gathered}
\sup _{z \in T}\left\|X_{j}^{0}(z)\right\|_{k, p} \leqslant C, \\
\sup _{0<\varepsilon \leqslant 1} \sup _{z \in T} \mathrm{E}\left(\left|X_{j}^{\varepsilon}(z)\right|^{p}\right) \leqslant C, \\
\sup _{0<\varepsilon \leqslant 1} \sup _{z \in T} \sup _{\boldsymbol{\alpha}: \sup \boldsymbol{\alpha}<z} \mathrm{E}\left(\left|D_{\boldsymbol{\alpha}}^{k} X_{j}^{\varepsilon}(z)\right|^{p}\right) \leqslant C,
\end{gathered}
$$

for any $j, k \in \mathbb{N}, p \in(1, \infty)$ and some positive constant $C$.

From the remark following Lemma 3.2 , one clearly gets $X_{j}^{0}(z) \in \mathscr{H}_{j}$, for any $j \in \mathbb{N}$. This yields (3.10).

We know (Rovira and Sanz-Solé 1996) that

$$
\sup _{0<\varepsilon \leqslant 1} \sup _{z \in T} \mathrm{E}\left(\left|X_{z}^{\varepsilon}\right|^{p}\right) \leqslant C, \quad p \in(1, \infty) .
$$

Then, the standard arguments based on the Burkholder, the Hölder and the Gronwall inequalities applied to (3.5) and (3.6) yield (3.11) by a recursive argument.

Finally, for the proof of (3.12) we first write the equations satisfied by $D_{\boldsymbol{\alpha}}^{k} X_{j}^{\varepsilon}(z), j \in \mathbb{N}$; this can be done using (3.5), (3.6) and the rules of Malliavin calculus. Then we proceed as for the proof of (3.11). This estimate allows use of the recursive argument which is needed.

We finish the study of this example by checking the uniform non-degeneracy property. We need the following additional assumptions on the coefficients:

(H3) $\left|a_{3 j}(s, t)-a_{3 j}\left(s^{\prime}, t^{\prime}\right)\right| \leqslant C\left\{\left|s-s^{\prime}\right|+\left|t-t^{\prime}\right|\right\}$,

$$
j=1,2,(s, t),\left(s^{\prime}, t^{\prime}\right) \in T,
$$

(H4) $\sup _{t \in[0,1]}\left|a_{4 j}(s, t)-a_{4 j}\left(s^{\prime}, t\right)\right| \leqslant C\left|s-s^{\prime}\right|, \quad j=1,2,\left(s, s^{\prime}\right) \in T$, $\sup _{(s, t) \in T}\left|\partial_{1}^{2} a_{3 j}(s, t)\right| \leqslant C, \quad j=1,2$,

(H5) $a_{31}(0, t) x_{0}+a_{32}(0, t) \neq 0, \quad t \neq 0$,

(H6) $a_{31}(0, v) x_{0}+a_{32}(0, v)=0, \quad \forall v \in(0, t]$, $\partial_{1} a_{31}(0, t) x_{0}+\partial_{1} a_{32}(0, t)+a_{31}(0, t) \int_{0}^{t} \gamma_{0, t}(0, w)\left\{a_{41}(0, w) x_{0}+a_{42}(0, w)\right\} \mathrm{d} w \neq 0$, for some positive constant $C$ and where $\partial_{1}$ means the derivative with respect to the variable $s$. 
Proposition 3.5 of Rovira and Sanz-Solé (1996) establishes $X_{s, t} \in \mathbb{D}^{\infty}$ under (H1) and (H2), for any $(s, t) \in T$.

Proposition 3.4. Let $z=(s, t) \in T$, st $\neq 0$ be fixed. One of the following set of conditions implies that $\left\|\Gamma_{X_{z}^{\varepsilon}}^{-1}\right\|_{p} \leqslant C \varepsilon^{-2}$, for some positive constant $C$ and every $\varepsilon \in(0,1], p \in(1, \infty)$ :

(a) (H1) to (H3) and (H5);

(b) (H1) to (H4) and (H6).

Proof. It suffices to check that the inverse of the random variable $\varepsilon^{-2} \int_{R_{z}}\left|D_{\alpha} X_{z}^{\varepsilon}\right|^{2} \mathrm{~d} \alpha$ has moments of any order. Consider the stochastic differential equation

$$
Y_{z}^{\varepsilon}(\alpha)=\gamma_{z}(\alpha)+\int_{(\alpha, z]} \gamma_{z}(\eta) Y_{\eta}^{\varepsilon}(\alpha)\left\{\varepsilon a_{3,1}(\eta) \mathrm{d} W_{\eta}+a_{4,1}(\eta) \mathrm{d} \eta\right\}, \quad 0 \leqslant \alpha \leqslant z .
$$

Then, $D_{\alpha} X_{z}^{\varepsilon}=\varepsilon a_{3}\left(X_{\alpha}^{\varepsilon}, \alpha\right) Y_{z}^{\varepsilon}(\alpha)$. Consequently we need to show that

$$
P\left(\int_{R_{z}}\left\{a_{3}\left(X_{\alpha}^{\varepsilon}, \alpha\right) Y_{z}^{\varepsilon}(\alpha)\right\}^{2} \mathrm{~d} \alpha \leqslant \eta\right) \leqslant \eta^{p},
$$

for any $p \in(1, \infty)$ and $\eta \leqslant \eta_{0}$.

This has been proved in Propositions 3.6 and 3.7 of Rovira and Sanz-Solé (1996). We point out that although assumptions (H3) and (H4) in this reference are stronger, they can be relaxed to the situation of our statement.

Propositions 3.1, 3.3 and 3.4 establish all the necessary ingredients to apply Theorem 2.4 to the family $\left\{X_{z}^{\varepsilon}, \varepsilon \in(0,1]\right\}$ defined by (3.3) with $z=(s, t) \in T$, st $\neq 0$.

\subsection{An Itô equation on the plane}

Consider a one-dimensional Wiener process $\left\{W_{s, t},(s, t) \in T\right\}, T=[0,1]^{2}$, vector fields

$$
A(x)=\left(\begin{array}{l}
x_{1} \\
x_{2}
\end{array}\right), \quad A_{0}(x)=\left(\begin{array}{c}
x_{2} \\
x_{1}
\end{array}\right)
$$

and the stochastic differential equation on $\mathbb{R}^{2}$ :

$$
Z_{z}=x_{0}+\int_{R_{z}}\left\{A\left(Z_{\eta}\right) \mathrm{d} W_{\eta}+A_{0}\left(Z_{\eta}\right) \mathrm{d} \eta\right\}, \quad z \in T,
$$

with initial condition $X_{0}=\left(\begin{array}{l}1 \\ 0\end{array}\right)$.

Let $\left\{Z_{z}^{\varepsilon}, z \in T\right\}$ be the solution of

$$
Z_{z}^{\varepsilon}=x_{0}+\int_{R_{z}}\left\{\varepsilon A\left(Z_{\eta}^{\varepsilon}\right) \mathrm{d} W_{\eta}+A_{0}\left(Z_{\eta}^{\varepsilon}\right) \mathrm{d} \eta\right\}
$$

and $\{\Psi(z), z \in T\}$ be given by 


$$
\Psi(z)=x_{0}+\int_{R_{z}} A_{0}(\Psi(\eta)) \mathrm{d} \eta
$$

In the sequel, $z$ will be a fixed point in $T$ not on the axis. The analogue of Proposition 3.1 for the solution of (3.13) can be proved by the same arguments, owing to the linearity of the coefficients $A$ and $A_{0}$. Thus,

$$
Z_{z}^{\varepsilon}=\mathrm{E} Z_{z}+\sum_{n=1}^{\infty} \varepsilon^{n} I_{n}\left(f_{n}\right)
$$

where $Z_{z}=\mathrm{E} Z_{z}+\sum_{n=1}^{\infty} I_{n}\left(f_{n}\right)$ is the Wiener-chaos decomposition of the $L^{2}$-functional $Z_{z}$.

Let $\hat{Z}_{z}^{\varepsilon}=\left\{Z_{z}^{\varepsilon}-\Psi(z)\right\} / \varepsilon$. Following the ideas of the proof of Proposition 3.3 we obtain

$$
\sup _{0<\varepsilon \leqslant 1}\left\|\frac{\mathrm{d}^{j}}{\mathrm{~d} \varepsilon^{j}} \hat{Z}_{z}^{\varepsilon}\right\|_{k, p} \leqslant C,
$$

for any $j \in Z^{+}, k \in \mathbb{N}, p \in(1, \infty)$.

In Nualart and Sanz-Solé (1989) we have proved that $Z_{z} \in \mathbb{D}^{\infty}$ and $\left\|\Gamma_{Z_{z}}^{-1}\right\|_{p} \leqslant C$, for any $p \in[1, \infty)$. By considering the coefficient $\varepsilon A$ instead of $A$ we have $\left\|\Gamma_{Z^{\varepsilon}}^{-1}\right\|_{p} \leqslant C_{\varepsilon}$, $p \in[1, \infty)$, for some constant $C_{\varepsilon}$ depending on $\varepsilon \in(0,1]$. Moreover, the Malliavin derivative of $Z_{z}^{\varepsilon}$ satisfies the stochastic differential equation

$$
D_{\alpha} Z_{z}^{\varepsilon}=A\left(Z_{\alpha}^{\varepsilon}\right)+\int_{(\alpha, z]}\left\{\varepsilon \nabla A\left(Z_{\eta}^{\varepsilon}\right) D_{\alpha} Z_{\eta}^{\varepsilon} \mathrm{d} W_{\eta}+\nabla A_{0}\left(Z_{\eta}^{\varepsilon}\right) D_{\alpha} Z_{\eta}^{\varepsilon} \mathrm{d} \eta\right\} .
$$

We close this section by checking that $\left\|\Gamma_{Z_{z}^{\varepsilon}}^{-1}\right\|_{p} \leqslant C \varepsilon^{-2}$ for any $\varepsilon \in(0,1], p \in(1, \infty)$ and some positive constant $C$. Clearly, it suffices to show that

$$
\sup _{\varepsilon \in(0,1]} \mathrm{E}\left(\left|\operatorname{det} \gamma_{\varepsilon}^{-1}\right|^{p}\right) \leqslant C, \quad p \in(1, \infty)
$$

with $\gamma_{\varepsilon}=\varepsilon^{-2} \Gamma_{Z_{z}^{\varepsilon}}$. This property will follow from

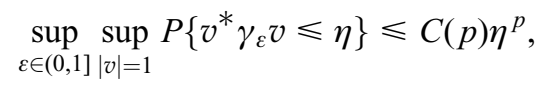

for any $p \in(1, \infty)$ and $\eta$ small enough. Using (3.14) we easily obtain

$$
\gamma_{\varepsilon}^{i j}=\int_{R_{z}} \xi_{k}^{\varepsilon, i}(z, r) A^{k}\left(Z_{r}^{\varepsilon}\right) \xi_{k^{\prime}}^{\varepsilon, j}(z, r) A^{k^{\prime}}\left(Z_{r}^{\varepsilon}\right) \mathrm{d} r, \quad 1 \leqslant i, j \leqslant 2,
$$

where $\left\{\xi^{\varepsilon}(z, r), 0 \leqslant r \leqslant z\right\}$ is an $\mathbb{R}^{2} \otimes \mathbb{R}^{2}$-valued process solution to the stochastic differential equation

$$
\xi^{\varepsilon}(z, r)=I+\int_{(r, z]}\left\{\varepsilon \nabla A\left(Z_{\eta}^{\varepsilon}\right) \xi^{\varepsilon}(\eta, r) \mathrm{d} W_{\eta}+\nabla A_{0}\left(Z_{\eta}^{\varepsilon}\right) \xi^{\varepsilon}(\eta, r) \mathrm{d} \eta\right\} .
$$

Then, as in Nualart and Sanz-Solé (1989), the proof of (3.15) is reduced to that of

$$
\sup _{\varepsilon \in(0,1]|r|=1} \sup _{|r|=1} P\left\{\int_{0}^{s}\left|v_{i} A^{i}\left(Z_{\sigma t}^{\varepsilon}\right)\right|^{2} \mathrm{~d} \sigma \leqslant \eta\right\} \leqslant C(p) \eta^{p} .
$$


Let $\mathscr{D}=\left\{A, A_{0}^{\nabla} A\right\}$ where $A_{0}^{\nabla} A$ denotes the covariant derivative of $A$ in the direction of $A_{0}$. Clearly the span of $\mathscr{D}$ at

$$
X_{0}=\left(\begin{array}{l}
1 \\
0
\end{array}\right)
$$

is $\mathbb{R}^{2}$. Consequently, there exists $R>0, c>0$, such that

$$
\sum_{V \in \mathscr{D}}\left\{v_{i} V^{i}(y)\right\}^{2} \geqslant c
$$

for any $|v|=1$ and $y \in B_{R}\left(x_{0}\right)$.

Let $S^{\varepsilon}=\inf \left\{\sigma \geqslant 0: \sup _{\xi \leqslant \sigma, \tau \leqslant t}\left|Z_{\xi \tau}^{\varepsilon}-x_{0}\right| \geqslant R\right\} \wedge s$. Then

$$
P\left(\int_{0}^{S^{\varepsilon}}\left|v_{i} A^{i}\left(Z_{\sigma t}^{\varepsilon}\right)\right|^{2} \mathrm{~d} \sigma<\eta\right) \leqslant p_{1}^{\varepsilon}(\eta)+p_{2}^{\varepsilon}(\eta)+p_{3}^{\varepsilon}(\eta),
$$

with

$$
\begin{aligned}
& p_{1}^{\varepsilon}(\eta)=P\left(\int_{0}^{S^{\varepsilon}}\left|v_{i} A^{i}\left(Z_{\sigma t}^{\varepsilon}\right)\right|^{2} \mathrm{~d} \sigma<\eta, \int_{0}^{S^{\varepsilon}}\left|v_{i}\left(A_{0}^{\nabla} A\right)^{i}\left(Z_{\sigma t}^{\varepsilon}\right)\right|^{2} \mathrm{~d} \sigma<\eta^{\alpha}, S^{\varepsilon} \geqslant \eta^{\beta}\right), \\
& p_{2}^{\varepsilon}(\eta)=P\left\{S^{\varepsilon}<\eta^{\beta}\right\}, \\
& p_{3}^{\varepsilon}(\eta)=P\left(\int_{0}^{S^{\varepsilon}}\left|v_{i} A^{i}\left(Z_{\sigma t}^{\varepsilon}\right)\right|^{2} \mathrm{~d} \sigma<\eta, \int_{0}^{S^{\varepsilon}}\left|v_{i}\left(A_{0}^{\nabla} A\right)^{i}\left(Z_{\sigma t}^{\varepsilon}\right)\right|^{2} \mathrm{~d} \sigma \geqslant \eta^{\alpha}\right),
\end{aligned}
$$

where $0<\beta<\alpha<1$.

Property (3.16) and the choice of $\beta$, $\alpha$ yields $p_{1}^{\varepsilon}(\eta)=0$ for $\eta$ sufficiently small. The Chebyshev inequality together with the Burkholder and the Hölder inequalities ensure that $\sup _{\varepsilon \in(0,1]} p_{2}(\eta) \leqslant C \eta^{\beta q / 2}$. The term $p_{3}^{\varepsilon}(\eta)$ demands a careful analysis. This has been done by Nualart and Sanz-Solé $\left(1989\right.$, p. 15) and corresponds to the term $A_{2}$ in this reference with $V=A, X_{\sigma} \cdot=Z_{\sigma_{\dot{\nabla}}}^{\varepsilon} \varepsilon^{m(j-1)}=\eta, \alpha=m(j) / m(j-1)$. As a hint for the reader, we point out that span $\left(A\left(x_{0}\right), A_{0}^{\nabla} A\left(x_{0}\right)\right)=\mathbb{R}^{2}$ implies the validity of assumption (H2) of Theorem 2.2 of Nualart and Sanz-Solé (1989). Indeed, using the notation in this article,

$$
A\left(x_{0}\right)=A^{1}\left(x_{0}\right), \quad A_{0}^{\nabla} A\left(x_{0}\right)=\left(\int_{0}^{1}\left(A_{0} * A\right)(\tau, 1) \mathrm{d} \tau\right)\left(x_{0}\right) .
$$

Since all the estimates in the above-mentioned proof can be obtained uniformly in the parameter $\varepsilon$, we conclude. 


\section{Acknowledgements}

The authors thank Professor G. Ben Arous and Professor D. Nualart for some helpful remarks. Thanks are also due to the referees for their comments. This work has been partially supported by Grant PB 930052 from the Dirección General de Investigación Certífica y Técnica and Grant CT060075/EU from the European Union.

\section{References}

Aida, S., Kusuoka, S. and Stroock, D. (1993) On the support of Wiener functionals. In K.O. Elworthy and N. Ikeda (eds), Asymptotic Problems in Probability Theory: Functionals and Asymptotics, pp. 3-34. Pitman Res. Notes Math. 284. London: Longman.

Azencott, R. (1984) Densités des diffusions en temps petit: développements asymptotiques. Séminaire de Probabilités XVIII 1982-1983, pp. 402-498. Lecture Notes Math. 1059. Berlin: SpringerVerlag.

Ben Arous, G. (1988) Méthodes de Laplace et de la phase stationnaire sur l'espace de Wiener. Stochastics, 25, 125-153.

Bismut, J.M. (1984) Large Deviations and the Malliavin Calculus. Prog. Math. 45. Basel: Birkhäuser.

Farré, M. and Nualart, D. (1993) Nonlinear stochastic integral equation in the plane. Stochastic Processes Applic., 46, 219-239.

Léandre, R. (1988) Applications quantitatives et géometriques du calcul de Malliavin. In M. Metivier and S. Watanabe (eds), Stochastic analysis, Proceedings, Paris 1987, pp. 109-133. Lecture Notes Math. 1322. Berlin: Springer-Verlag.

Léandre, R. and Russo, F. (1992) Small stochastic perturbation of a one-dimensional wave equation. In H. Korezlioglu and S. Ustunel (eds), Stochastic Analysis and Related Topics, pp. 285-332. Prog. Probab. 31. Boston Basel Berlin: Birkhäuser.

Molchanov, S.A. (1975) Diffusion processes and Riemannian geometry. Russian Math. Surveys, 30, $1-63$.

Nualart, D. (1995) Malliavin Calculus and Related Topics. Berlin: Springer-Verlag.

Nualart, D. (1998) Analysis on Wiener space and anticipating stochastic calculus. École d'été de Probabilités de Saint-Flour XXV, pp. 123-227. Lecture Notes Math. 1690. Berlin: SpringerVerlag.

Nualart, D. and Sanz-Solé, M. (1989) Stochastic differential equations on the plane: smoothness of the solution. J. Multivariate Anal., 31, 1-29.

Rovira, C. and Sanz-Solé, M. (1996) The law of the solution to a nonlinear hyperbolic SPDE. J. Theor. Probab., 9, 863-901.

Stroock, D.W. (1987) Homogeneous chaos revisited. Séminaire de Probabilités XXI, pp. 1-8. Lecture Notes Math. 1247 Berlin: Springer-Verlag.

Takanobu, S. and Watanabe, S. (1993) Asymptotic expansion formulas of the Schilder type for a class of conditional Wiener functional integrations. In K.O. Elworthy and N. Ikeda (eds), Asymptotic Problems in Probability Theory: Functionals and Asymptotics, pp. 194-241. Pitman Res. Notes Math. 284. London: Longman.

Watanabe, S. (1987) Analysis of Wiener functionals (Malliavin calculus) and its applications to heat kernels. Ann. Probab., 15, 1-39.

Received March 1997 and revised September 1997 